\title{
Christian Methfessel, Kontroverse Gewalt: Die Imperiale Expansion in der englischen und deutschen Presse vor dem Ersten Weltkrieg
}

\section{Sébastien Tremblay}

\section{OpenEdition}

1 Journals

Electronic version

URL: https://journals.openedition.org/ifha/11092

DOI: 10.4000/ifha.11092

ISSN: 2198-8943

Publisher

IFRA - Institut franco-allemand (sciences historiques et sociales)

Electronic reference

Sébastien Tremblay, "Christian Methfessel, Kontroverse Gewalt: Die Imperiale Expansion in der englischen und deutschen Presse vor dem Ersten Weltkrieg", Revue de I'IFHA [Online], Date of review, Online since 26 January 2021, connection on 11 October 2021. URL: http://journals.openedition.org/ifha/11092 ; DOI: https://doi.org/10.4000/ifha.11092

This text was automatically generated on 11 October 2021.

(CIFHA 


\section{Christian Methfessel, Kontroverse Gewalt: Die Imperiale Expansion in der englischen und deutschen Presse vor dem Ersten Weltkrieg}

Sébastien Tremblay

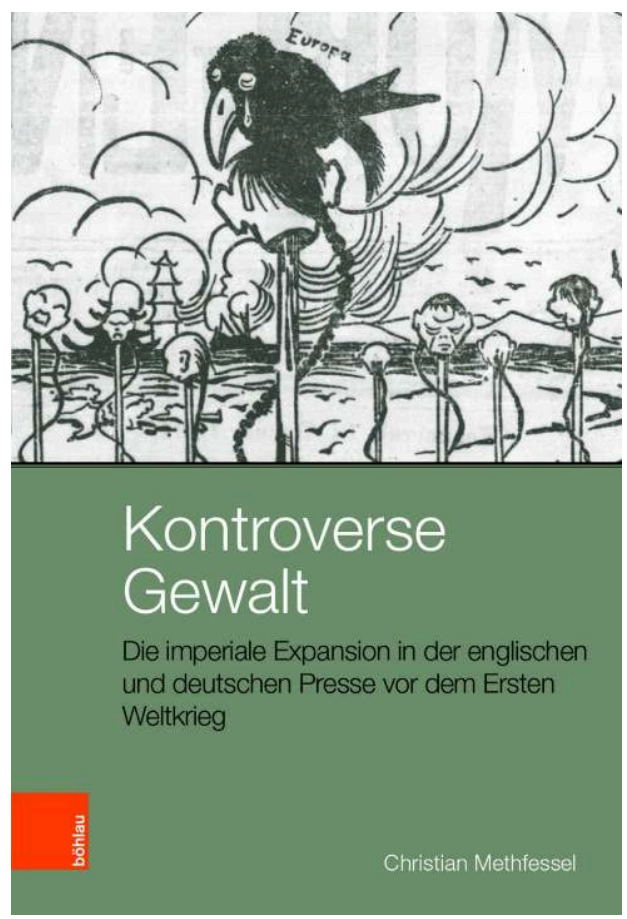

La reconstruction de l'ancien palais prussien au cœur de Berlin, aujourd'hui le Humboldt Forum, a relancé plusieurs débats sur la nostalgie de la période Wilhelmienne et sur le colonialisme allemand. Dans cet ouvrage, Christian Methfessel, historien à l'Université d'Erfurt, offre une importante contribution à ces discussions. 
Étudiant simultanément la presse écrite allemande à l'époque Guillaume II et son équivalence britannique, il propose une analyse minutieuse de son rôle discursif lors des débats coloniaux au tournant du XXe siècle. Effectivement, l'auteur démontre clairement les différentes tensions au sein de la presse écrite au sujet de l'expansion coloniale anglaise et allemande du siècle dernier. En analysant un riche corpus de journaux, tantôt conservateurs, tantôt libéraux, parfois ouvriers, il réussit à souligner les critiques face à l'expansion coloniale, rompant ici avec l'idée préconçue d'un paysage médiatique uni autour de la question. Comme il le met en évidence à maintes reprises, ces critiques (basées sur des bases politiques, pécuniaires et parfois opportunes) ne doivent pas être confuses avec une délégitimation du système colonial ou des hiérarchies raciales européennes de l'époque. Contribuant à une nouvelle historiographie impériale et liant une histoire des médias aux méthodologies de l'histoire globale, Methfessel ancre son analyse dans une histoire européenne, tout en montrant la puissance discursive des évènements hors Europe, le rôle de la presse comme vecteur communicatif et les considérations capitalistes des lignes éditoriales de l'époque. Signalant le rôle historique des médias pour le pouvoir en place, en tant que critique ou en tant que héraut, il montre à quel point la création des médias de masse chamboulèrent les normes du début du XXe siècle (p. 133-141). De plus, Kontroverse Gewalt démontre l'apport des médias aux perspectives coloniales de la métropole, en soulignant leur connexion à l'essor du télégraphe et à la prise en compte de certain-es acteurs et actrices historiques. Par exemple, l'auteur met l'accent sur les lettres de soldats publiés par la presse, notamment durant la violence génocidaire du pouvoir colonial allemand en Afrique (p. 282), le rôle joué par les missionnaires allemands en Chine (p. 153-154) ainsi que sur la dominance britannique dans le domaine des correspondants étrangers, notamment les tensions entre l'Allemagne et l'Angleterre vers la fin de la période mise en lumière dans son livre (p. 293).

L'ouvrage est structuré chronologiquement et thématiquement. Les trois chapitres principaux offrent un examen détaillé de l'évolution des guerres et crises coloniales anglaises et allemandes entre 1896 et 1911. Ces différents tableaux ne font pas que comparer une pluralité de journaux, mais démontrent également les liens étroits entre les deux métropoles, surtout en examinant les incidents diplomatiques ayant envenimés les relations anglo-allemandes, par exemple l'animosité déclenchée par le support de Guillaume II aux Boers. Methfessel pointe avec brio la discordance entre relations tendues au niveau des états et le caractère venimeux des attaques médiatiques de part et d'autre de la Manche. En effet, il démontre à quel point les journaux des deux pays n'étaient pas toujours au diapason des tensions diplomatiques. Le premier chapitre souligne l'enthousiasme plutôt généralisé de la presse pour l'expansion coloniale en soulignant l'intervention britannique au Soudan ou l'annexion de la concession de Kiautschou par l'état allemand. Le deuxième chapitre démontre un point tournant autour de 1899-1902 et utilise entre autre la seconde guerre des Boers et la guerre des Boxers pour exemplifier les nouvelles perceptions de l'expansion coloniale dans la presse des deux pays. Ce chapitre met en lumière la dégradation de l'image de l'Empire allemand dans la presse écrite anglaise et vice-versa (p. 196). Le troisième et dernier chapitre met l'accent sur la valeur accordée aux crises coloniales dans les médias (Deutungsrahmenanalyse) et illustre l'importance et l'instrumentalisation de l'outre-mer colonial pour la politique domestique des deux empires. À ces trois chapitres sont liés trois autres analyses thématiques. Une première décrit l'essor des médias de masse comme le Daily Mail en Angleterre et le Berlin Lokal- 
Anzeiger et leurs influences sur le politique et la diplomatie. La deuxième se concentre sur l'aspect global de la couverture médiatique analysée. Cette partie est particulièrement louable pour les liens tissés par l'auteur entre la manière qu'avait l'Europe de se percevoir dans un contexte intra-Européen ou mondial (Selbstbild et Fremdbild) et le lien entre mission civilisatrice, hiérarchies raciales, prosélytisme colonial, présumée supériorité et perceptions de l'ennemi non-Européen résistant. Finalement la dernière partie démontre que les modèles de légitimation du débat politique avaient leur propre logique, étroitement liée aux formes spécifiques de représentation des médias de masse. Methfessel examine ici les liens entre critiques de certaines guerres coloniales et démontre que plus souvent qu'autrement, le coût des opérations ou les répercussions politiques au sein de la métropole jouait un rôle plus important que la violence coloniale en soit.

Plusieurs trouveront ici non seulement une étude hors-pair, mais également une mine de sources pour leurs propres recherches. Le livre n'est cependant pas sans bémol. Premièrement, le choix de l'auteur de considérer chaque périodique comme un seul acteur historique suivant une ligne éditoriale précise (p. 19) semble plutôt opportun pour son argumentaire. Même s'il est vrai que plusieurs articles de l'époque ne sont pas signés et que la majorité des journaux avaient une ligne directrice, la contestation au sein des rédactions aurait pu être explorée. Deuxièmement, malgré la mention d'une méthodologie liée à l'histoire globale de plus en plus pratiquée en Allemagne, les transferts décrits par l'auteur semblent plus souvent conçus dans une logique 'centre et périphéries' que dans une logique d'enchevêtrement. Finalement, il aurait était intéressant de porter plus attention aux images dans les journaux étudiés. Outre certaines caricatures, l'auteur rejette rapidement une analyse visuelle de ses sources ( $p$. 352), signifiant que la plupart des images publiées représentaient des paysages et non pas des guerres ou conflits. L'auteur doit pourtant savoir que tout un pan de l'historiographie allemande des concepts aurait pu lui permettre d'analyser la conceptualisation de ces images. En ce sens, un focus unique sur le texte semble ici un choix qui aurait pu être mieux défendu. Somme toute, Methfessel offre un livre qui servira à plusieurs. Le nombre impressionnant de sources lié à son analyse méticuleuse est une solide contribution à l'histoire coloniale et l'histoire des médias à l'aube de la Grande Guerre.

\section{INDEX}

Subjects: Histoire des États et des pouvoirs, Histoire des idées

Chronological index: Epoque contemporaine

\section{AUTHOR}

\section{SÉBASTIEN TREMBLAY}

Freie Universität Berlin, Friedrich-Meinecke-Institut, Arbeitsbereich Globalgeschichte 